\title{
Biocomercio, comercio justo y asociatividad modelo para los superfoods peruanos
}

EduARdo Buse THORNE ${ }^{1}$ Mario Chong ChONG ${ }^{2}$ LuZ VÍLCHEZ TÚPAC

\section{INTRODUCCIÓN}

En los últimos años, se ha reflexionado y prestado mayor atención a las alternativas de valor agregado a nuestras exportaciones, con el desarrollo de cadenas productivas bajo la intervención del capital humano y el conocimiento de manera intensiva, en busca de una estrategia para reducir la pobreza y contribuir al desarrollo sostenible.

Sin duda, el biocomercio es un componente importante en ese sentido. El Perú es uno de los países con mayor biodiversidad en el mundo y, paradójicamente, esa riqueza natural está ubicada en las regiones y poblaciones más pobres del país. Sin embargo, las experiencias exitosas están aumentando, con una mejora significativa en el nivel de vida de muchos compatriotas como resultado de esfuerzos individuales, asociativos, casi sobrehumanos y sin recursos (INDECOPI, 2016).

Por su gran biodiversidad, el Perú posee importantes cantidades de recursos naturales, entre los cuales destaca la maca (Lepidium Meyeni), domesticada durante milenios por los antepasados de los incas (INDECOPI, 2014).

Los pobladores Pumpush, cultura pre-inca de las zonas de Cuncush Runa- Junín, fueron los primeros en cultivar y consumir regularmente la maca. Esta raíz crece sobre los 4000 m.s.n.m. altitud donde no prospera ningún otro cultivo debido a la combinación de condiciones extremas de frio, fuertes vientos y alta radiación solar (Hermann y Bernet, 2009).

Las plantas ancestrales de la maca habrían sido una de sus fuentes de alimento, y el proceso de su domesticación se habría iniciado 1200 años A.C. en los alrededores de San Blas, en el lugar denominado Pacha-Machay. Otros indicios demuestran que hace 2000 años se consumía carne de camélidos y maca (como raíz silvestre).

Este alimento milenario se presenta como alimento nutritivo y energético, convirtiéndose en una alternativa de desarrollo para las comunidades de las zonas altas de la Cordillera de los Andes de Perú.

1 PhD en Gestión de Empresas por la UNMSM, profesor de la Escuela de Posgrado de la Universidad del Pacífico. ew.buset@up.edu.pe

$2 \mathrm{PhD}$ en Gestión de Empresas por la UNMSM, Vicedecano de Ingeniería Empresarial de la Universidad del Pacífico. m.chong@up.edu.pe

3 Egresada de la maestría en Agronegocios y Alimentos de la Universidad del Pacífico. 1.vilcheztupac@alum.up.edu.pe 
La denominación de origen "Maca Junín - Pasco" del año 2011 por el Instituto Nacional de Defensa de la Competencia y de la Protección de la Propiedad Intelectual (INDECOPI, 2014), logró consolidar su mercado por la zona de producción y los métodos de extracción (INDECOPI, 2016). Actualmente, la maca es reconocida como un "Superalimento o Superfood" por sus propiedades funcionales y nutricionales, con el apoyo del Estado Peruano (PROMPERU, 2017).

El problema es ¿Cómo lograr la sinergia entre el biocomercio, agricultura orgánica, comercio justo y la asociatividad para lograr que los superfoods peruanos sean exitosos en los mercados internacionales?

El objetivo del trabajo de investigación es elaborar un plan de desarrollo de los superfoods basado en el cultivo, transformación, comercialización y su articulación con el mercado internacional.

\section{MARCO TEÓRICO}

A fines de los años 80, inicia sus actividades PROMA$\mathrm{CA}^{4}$ cuya principal misión es promover y difundir el uso y consumo de la maca, debido a una situación preocupante: la casi inminente desaparición de la maca en el Perú. El proyecto consistía en incrementar el cultivo de maca e iniciar su comercialización. La producción, hasta ese momento, era solo para consumo familiar (PROMACA, 2017).

PROMACA inició sus actividades con la capacitación de los agricultores sobre prácticas agrícolas, desarrolló acciones de mantenimiento y cuidado de la fauna silvestre de vicuñas, desarrolló productos en base a la maca (jugo, refresco, harina y licor), participó en ferias locales, generó la difusión de sus propiedades a nivel nacional. En una segunda fase, promovió la producción orgánica y de comercio justo. Actualmente, la maca se exporta a mercados globales muy exigentes como Francia, Alemania, Suiza, Estados Unidos y Brasil.

La estrategia de PROMACA tienen relación con las tendencias del mercado y la demanda de productos con las siguientes características: libre de intolerancia, superalimentos con ingredientes naturales y orgánicos con propiedades funcionales y personalizados (Tolón y Lastra, 2009).

El mercado de productos orgánicos en el sector alimentos es uno de mayor crecimiento por la preocupación de los consumidores (SOAAN, 2013) (PROMPERU, 2014). Por este motivo la Comisión de Promoción del Perú para la Exportación y el Turismo (PROMPERU) realizó el lanzamiento mundial de la marca sectorial "Superfoods Perú", en la principal feria de alimentos, Fruit Logistics de Berlín, Alemania (PROMPERU, 2017).

La marca busca posicionar las propiedades nutricionales de los alimentos peruanos y una nueva fase en la promoción comercial de la oferta exportable. La estrategia de esta convocatoria, en el lado de la oferta, es el impulso de la asociatividad y sus prioridades competitivas: costo, calidad, tiempo, precio.

Los superfoods de esta nueva marca son: maca, kiwicha, cañihua, yacón, castaña, algarrobo, sacha inchi, maíz gigante del Cusco, maíz morado, camu camu, chirimoya, guanábana, lúcuma, mangos, uvas, mandarinas y anchoveta; además de los ya conocidos internacionalmente: arándanos, espárragos, brócoli, palta, granada, cacao y chocolates (PROMPERU, 2017). En el caso de la maca, el 80\% de la producción nacional se exporta a los Estados Unidos de Norte América, Europa y Asia (SUNAT, 2017).

La articulación con el mercado internacional, oferta y demanda, tiene tres componentes: biocomercio, comercio justo y asociatividad.

\section{BIOCOMERCIO}

El biocomercio o comercio sostenible de la biodiversidad es un modelo de negocio con tres pilares: la rentabilidad económica, la conservación de la biodiversidad y la consideración de aspectos sociales. El principal insumo es la biodiversidad nativa (PROMPERU, 2014) con actividades de recolección y/o producción, procesamiento y comercialización, e involucran la conservación y uso responsable de los recursos (UNCTAD, 2007).

Los enfoques de biocomercio pueden ser en cadena de valor, manejo adaptativo y ecosistémico (UNCTAD, 2007) con certificaciones orgánicas, de comercio justo y sociales, ambientales, sociales y de género.

\section{AGRICULTURA ORGÁNICA}

La agricultura orgánica es un sistema de gestión de la producción para optimizar la productividad y el equilibrio de las comunidades dentro del ecosistema, incluyendo suelo, plantas, ganado y personas; desarrollando empresas sostenibles y con armonía en el medio (Arbenz, Gould y Stopes, (2016) (Céspedes, 2005), para evitar la erosión, compactación, salinización y otras formas de degradación (Andersen, 2003).

4 Promotora Agroindustrial de Cultivos Andinos 
El mercado de productos orgánicos es uno de los de mayor crecimiento en el sector de alimentos, por la preocupación del consumidor en alimentarse saludablemente (SOAAN, 2013). Este tipo de agricultura tiene un componente humano muy importante con un sistema de tenencia de la tierra y la vinculación en los mercados.

\section{COMERCIO JUSTO}

El comercio justo tiene como objetivos mejorar el acceso a los mercados y brindar condiciones comerciales justas para los productores con un precio mínimo garantizado (Andersen, 2003).

El comercio justo es un sistema comercial basado en el diálogo, la transparencia y el respeto; busca una mayor equidad en el comercio internacional. Contribuye al desarrollo sostenible ofreciendo mejores condiciones comerciales y asegurando los derechos de los pequeños productores y trabajadores marginados, especialmente del sur. (Andersen, 2003, p. 13).

Los miembros de la Organización Mundial del Comercio Justo (WFTO) consideran toda la cadena productiva desde los proveedores hasta el cliente final y se rigen en diez principios: creación de oportunidades para productores en desventaja económica, transparencia y responsabilidad - rendición de cuentas, prácticas comerciales justas, pago de un precio justo, no al trabajo infantil y al trabajo forzoso, compromiso con la no discriminación, la igualdad de género y el empoderamiento económico de la mujer y la libertad de asociación, garantizar buenas condiciones de trabajo, desarrollo de capacidades, promoción del comercio justo y respeto por el medio ambiente (WFTO, 2017).

\section{ASOCIATIVIDAD}

La asociatividad es el resultado de la cooperación entre los agricultores y las empresas con el propósito de enfrentar la globalización y la competitividad de los mercados. Los indicadores en los trabajos realizados con camélidos sudamericanos (Buse y Chong, 2014), maca, chirimoya (Buse, Chong y Mathews, 2015) y cacao (Armas y Chong, 2016) muestran una mejora en la competitividad, productividad, rentabilidad, especialización y reducción del riesgo.

El componente fundamental en la asociatividad es el Comité de Desarrollo (CD) y sus ocho C (8C) del desarrollo (Buse y Chong, 2014): confianza, convivencia, credibilidad, conflictos, cantidad, calidad, continuidad y clima organizacional. Estos dos componentes, $C D \wedge 8 C$, generan las dimensiones del diamante del desarrollo (DD) con la moral y ética en el desempeño; el resultado es $\mathrm{DD}=f(\mathrm{CD} \wedge 8 \mathrm{C})$

\section{METODOLOGÍA Y RESULTADOS}

La sinergia entre el biocomercio, agricultura orgánica, comercio justo y la asociatividad para lograr que los superfoods peruanos sean exitosos en los mercados internacionales basados en el cultivo, transformación, comercialización y su articulación con el mercado internacional se puede lograr en tres fases desarrolladas por PROMACA.

La fase 1 - Biocomercio y agricultura orgánica: desde el año 2000, la empresa ha podido ingresar al mercado europeo, compensar la diferencia entre la producción orgánica (6 toneladas /hectárea) y la producción convencional (10 toneladas/hectárea) y reconocimiento a nivel internacional. Las figuras 1 y 2 (Vílchez, 2014) muestran las capacitaciones de PROMACA.

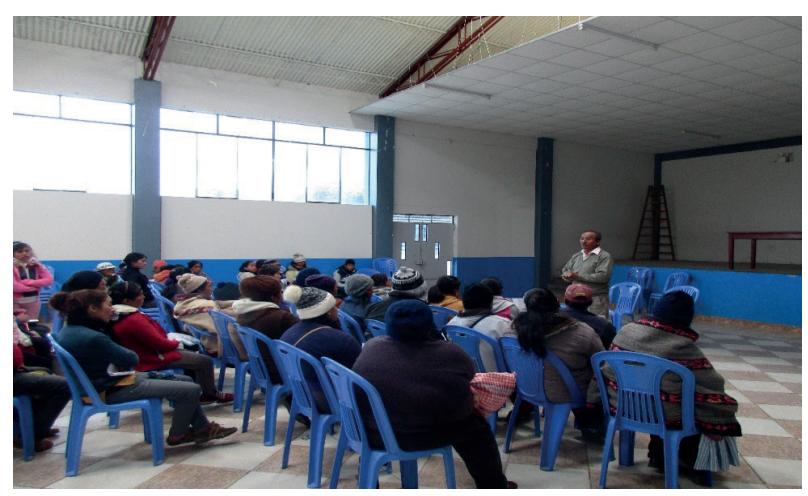

Figura 1: Capacitaciones sobre agricultura orgánica. Fuente: Vílchez, L. (2014).

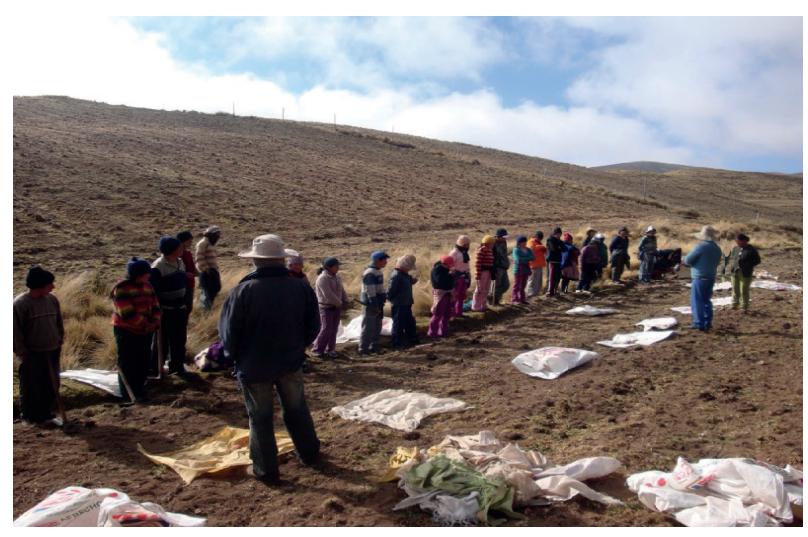

Figura 2: Capacitación sobre buenas prácticas agrícolas. Fuente: Vílchez, L. (2014). 
La fase 2 - Comercio justo: desde el año 2010 se han aplicado sus principios en todas las entidades (productores, trabajadores y la comunidad) y en el flujo para obtener el nivel Fair for Life. La figura 3 muestra el suministro de materiales de protección, la figura 4 (Vílchez, 2014) muestra la producción de maca y la figura 5 (Vílchez, 2014) la distribución de la maca procesada.

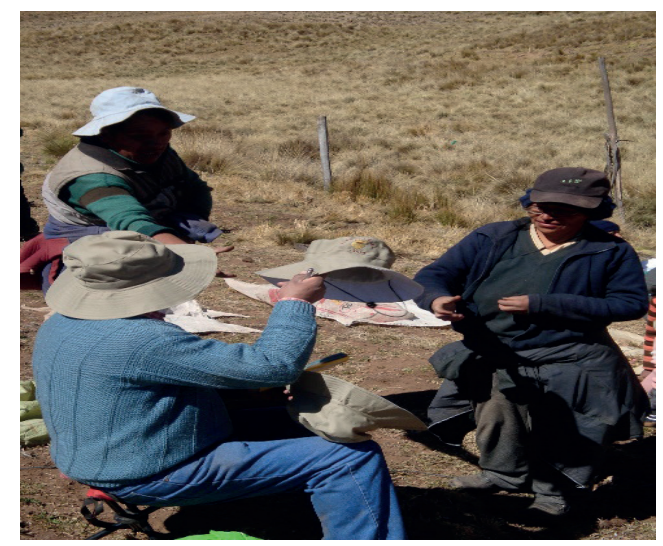

Figura 3. Entrega de materiales de protección. Fuente: Vílchez, L. (2014).

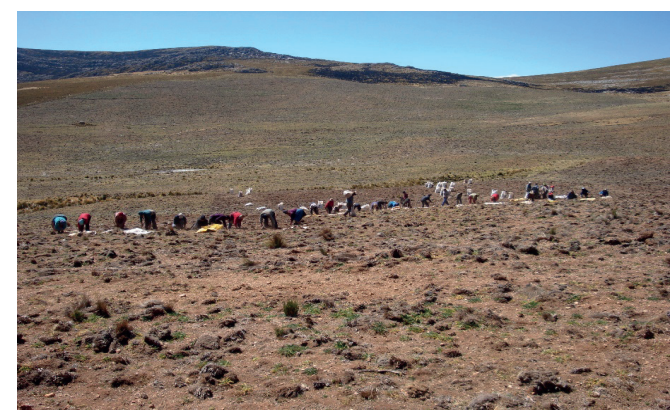

Figura 4. Cosecha de maca.

Fuente: Vílchez, L. (2014).

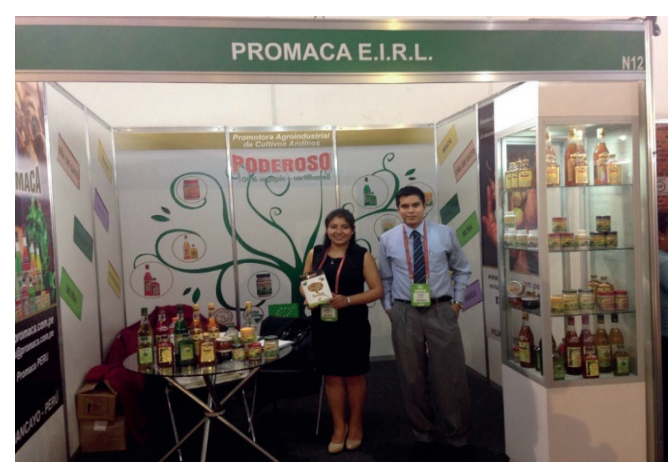

Figura 5. Comercialización de los productos en ferias. Fuente: Vílchez, L. (2014).
La fase 3 - Asociatividad: desde el año 2012 se enfoca en el diamante de desarrollo: $D D=f(C D \wedge$ $8 \mathrm{C})$, con la mejora de las condiciones de trabajo, vivienda, infraestructura, servicios médicos, desarrollo familiar e investigación en los procesos productivos. Uno de los proyectos es la mejora en el proceso de secado y la eliminación de micotoxinas. Las figuras 6 (Vílchez, 2014),7 (Vílchez, 2015) y 8 (Vílchez, 2016) muestran el impacto del programa.

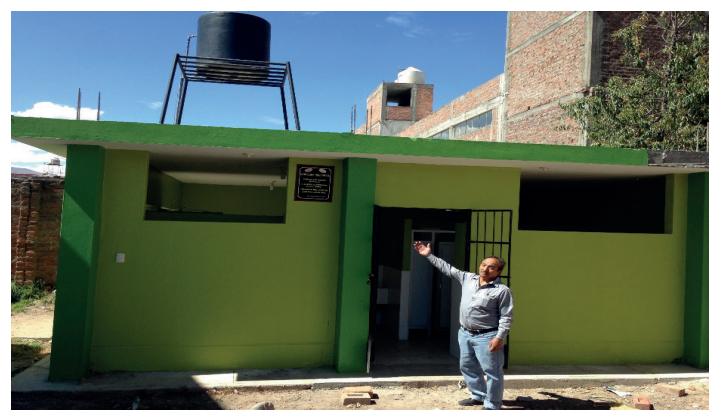

Figura 6. Donación de servicios higiénicos por la certificación de comercio justo.

Fuente: Vílchez, L. (2014).

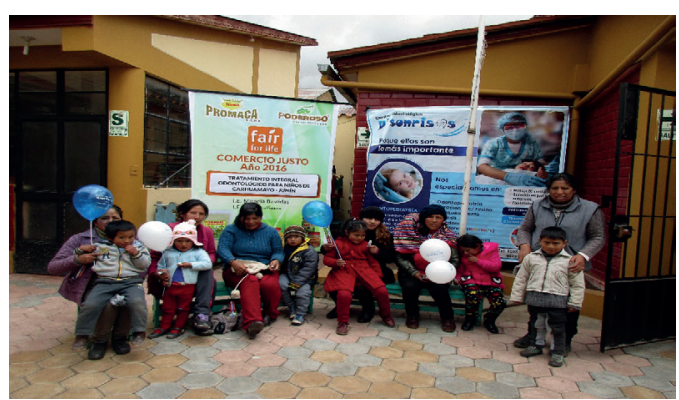

Figura 7. Beneficios para la comunidad por la certificación de comercio justo.

Fuente: Vílchez, L. (2015).

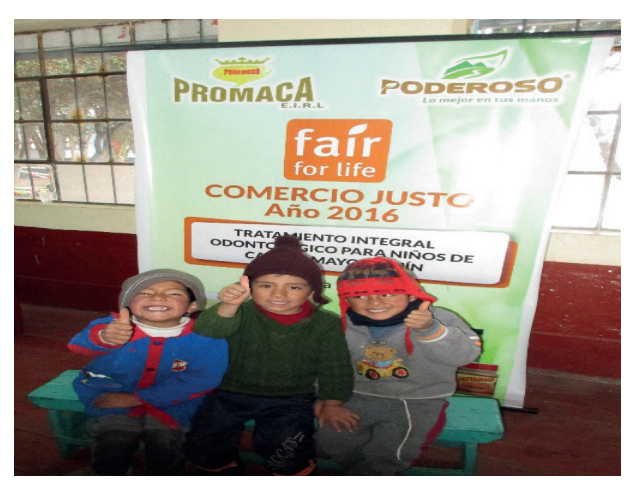

Figura 8. Tratamiento médico integral por la certificación de comercio justo en Carhuamayo.

Fuente: Vílchez, L. (2016). 
Las figuras 9 y 10 (Vílchez, 2016) muestran el impacto del proyecto con las investigaciones en el secado de la maca.

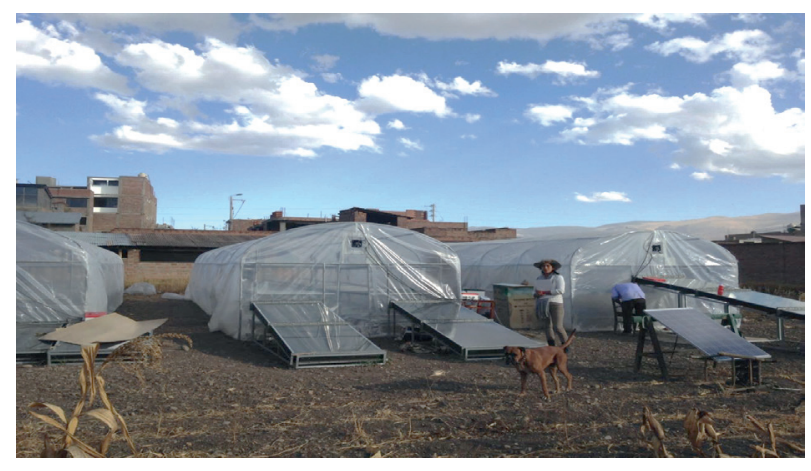

Figura 9. Proyecto de secado de maca, vista externa. Fuente: Vílchez, L. (2016).

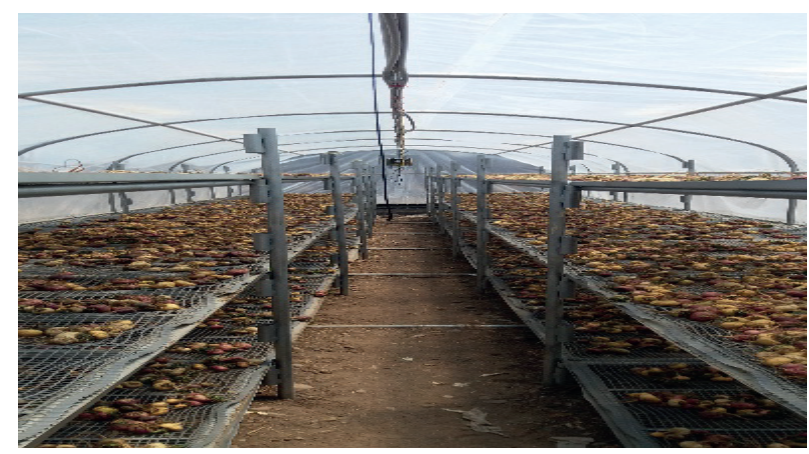

Figura 10. Proyecto de secado de maca, vista interna. Fuente: Vílchez. L. (2016).

La metodología ha permitido lograr un impacto responsable en la comunidad, con los procedimientos de la empresa de acuerdo a las normas internacionales de producción orgánica e inocuidad alimentaria (impacto ambiental), el reconocimiento de la maca a nivel mundial como un "superalimento", la valoración de su cultivo, la capacitación a los colaboradores en temas como agricultura orgánica, manejo agronómico responsable, asociatividad, entre otros acorde con las prácticas agrícolas (impacto social) y la optimización de la cadena de valor a precios justos en el merca internacional (impacto económico).

\section{DISCUSIÓN}

El factor confianza en las instituciones como pieza fundamental para la articulación en el mercado global de PROMACA tiene una fuerte relación con los estudios previos en el campo agrícola en Virú (Chong, 2012), la producción de chirimoya en Proachirko (Buse, Chong y Mathews, 2015), el modelo Biocunas de maca (Buse, Chong y Mathews, 2015) y el modelo Cadisa con el cacao (Armas y Chong, 2016).

La confianza es el fundamento para todas las transacciones sociales, económicas y emocionales (Global Scan, 2016). Estas relaciones de confianza se cultivan a largo plazo y está relacionada con la ética en los negocios. El rompimiento de la confianza impacta en tres dimensiones (Global Scan, 2016): credibilidad de la marca, caso United por aplicar revenue management; capital humano, caso Odebrecht por corrupción; y en su reputación, caso Volkswagen por manejo del software para el control de emisiones.

Los resultados del cuestionario radar de responsabilidad social (Global Scan, 2015) muestran un nivel medio de confianza de la población peruana en el gobierno con una media entre 45,48 sobre una base de 100, las grandes empresas peruanas y en las organizaciones no gubernamentales entre 50 y 56 de 100; las cuales impactan en el proceso de globalización de las empresas como PROMACA.

Los resultados del cuestionario radar 2015 sobre el nivel de confianza en el gobierno, las grandes empresas peruanas y las empresas globales operando en el Perú para actuar con el mejor interés para la sociedad tienen un nivel preocupante. Los valores asignados fueron 100 - confío mucho, 75 - confía algo, 50 - no confía mucho y 0 - no confía en absoluto.

Tabla 1. Nivel de confianza en las instituciones.

\begin{tabular}{|l|c|c|c|c|}
\hline & $\begin{array}{c}\text { Respuestas } \\
\text { válidas }\end{array}$ & Media & $\begin{array}{c}\text { Desviación } \\
\text { estándar }\end{array}$ & $\begin{array}{c}\text { Coeficiente } \\
\text { de } \\
\text { variación }\end{array}$ \\
\hline $\begin{array}{l}\text { Gobierno } \\
\text { nacional }\end{array}$ & 4945 & 45,48 & 32,759 & 72.03 \\
\hline $\begin{array}{l}\text { Grandes } \\
\text { empresas } \\
\text { peruanas }\end{array}$ & 4886 & 55,57 & 28,339 & 51.11 \\
\hline $\begin{array}{l}\text { Empresas } \\
\text { globales } \\
\text { operando } \\
\text { en el Perú }\end{array}$ & 4776 & 49,97 & 29,935 & 59.91 \\
\hline
\end{tabular}

Fuente: Global Scan. Cuestionario radar. (2015).

Los resultados muestran que no confían mucho en las entidades representativas para el desarrollo de los superfoods peruanos y el reto es mayor para organizaciones como PROMACA en un mercado global. 


\section{CONCLUSIONES}

El objetivo de este proyecto fue elaborar un plan de desarrollo para los Superfoods peruanos basado en el cultivo, transformación, comercialización y articulación con el mercado internacional.

El estudio realizado en PROMACA, empresa dedicada a la generación de valor de la maca, permitió establecer sus tres fases de desarrollo: biocomercio y agricultura orgánica, comercio justo y asociatividad.

Los resultados indican que la articulación con el mercado internacional se puede lograr con un ambiente cooperativo y la generación de confianza con las certificaciones; "...el modelo basado en la confianza es una alternativa para el aprovechamiento de las potencialidades que se pueda generar en beneficio de las diferentes regiones del Perú" (Buse, Chong y Mattews, 2015).

Los proyectos futuros deberían enfocarse en analizar otros grupos representativos y sus características. Adicionalmente, establecer las diferencias entre los grupos relacionados con la maca y analizar sus fases de desarrollo.

\section{REFERENCIAS BIBLIGRÁFICAS}

[1] Andersen, M. (2003). ¿Es la certificación algo para mí? Una guía práctica sobre por qué, cómo y con quién certificar productos agrícolas para la exportación. San José: Unidad Regional de Asistencia Técnica de la Organización de las Naciones Unidas para la Agricultura y la Alimentación (RUTA FAO).

[2] Arbenz, M., Gould, D., Stopes, C. (2016). Organic 3.0 - for truly sustainable farming and consumption. Bonn: IFOAM Organics International.

[3] Armas, D., Chong, M. (2016). Cadisa - Modelo de Desarrollo Inclusivo de las Pequeñas Unidades Agrícolas Ashaninkas, Perú. MedeIlín: Asamblea anual del Consejo Latinoamericano de Escuelas de Administración.

[4] Buse, E., Chong, M., Mathews, J. (2015). Modelo de gestión empresarial asociativo para PROACHIRKO, comunidad de Huanangui, Perú. Industrial Data. Vol. 18, Num. 2, 14-19.

[5] Buse, E., Chong, M., Mathews, J. (2015). Modelo de desarrollo para pequeños productores de maca en la región Junín, Perú. Valparaíso: Asamblea anual del Consejo Latinoamericano de Escuelas de Administración - CLADEA.
[6] Buse, E., Chong, M. (2014). Modelo de asociatividad para los criadores de camélidos sudamericanos del altiplano peruano. BarceIona: Asamblea anual del Consejo Latinoamericano de Escuelas de Administración - CLADEA.

[7] Céspedes, C. (2005). Agricultura orgánica. Principios y prácticas de producción. Chillán: Ministerio de Agricultura - Instituto de Investigaciones Agropecuarias.

[8] Chong, M. (2012). Talleres de asociatividad Diseño un modelo de gestión para el desarrollo de las pequeñas unidades agrícolas en el Perú. Industrial Data. Vol. 15, Num 1, 45-52.

[9] Comisión de Promoción del Perú para la Exportación y el Turismo - PROMPERU. (2014). Biocomercio: modelo de negocio sostenible. Lima: PROMPERU.

[10] Global Scan. (2015). Cuestionario radar de RSC. Lima: Global Scan.

[11] Global Scan. (2016). Trust in Global Companies. Toronto. Global Scan.

[12] Hermann,M., Bernet, T. (2009). The transition of maca from neglect to market prominence: Lesson for improving use strategies and market chains of minor crops. Rome: Agricultural Biodiversity and Livelihoods.

[13] Instituto Nacional de Defensa de la Competencia y de la Protección de la Propiedad Intelectual -INDECOPI. (2014). Boletín maca. Lima: Centro de información y documentación.

[14] Instituto Nacional de Defensa de la Competencia y de la Protección de la Propiedad Intelectual -INDECOPI. (2016). El registro de marca en el decreto legislativo $N^{\circ}$ 1075. Lima: Centro de información y documentación.

[15] Promotora Agroindustrial de Cultivos Andinos - PROMACA. (2017). Presentación de PROMACA. Lima. Recuperado de http://www.promaca.com.pe/paginaweb/inicio.php

[16] Superintendencia de Administración Tributaria - SUNAT. (2017). Estadística y estudios. Lima. Recuperado de http://www.sunat.gob. pe/estadisticasestudios

[17] Sustainable Organic Agriculture Action Network - SOAAN. (2013). Guía de mejores prácticas para la agricultura y cadenas de vaIor. Bonn: IFOAM Organics International.

[18] Tolón, A., Lastra, X. (2009). Los alimentos de calidad diferenciada. Una herramienta para el desarrollo rural sostenible. Almería: Universidad de Almería. 
[19] United Nations Conference on Trade and Development - UNCTAD. (2007). UNCTAD - Iniciativa BioTrade. Principios y criterios de biocomercio. Génova: ONU-UNCTAD.

[20] Vílchez, L. (2014). Capacitaciones sobre agricultura orgánica. Huancayo.

[21] Vílchez, L. (2014). Capacitación sobre buenas prácticas agrícolas. Huancayo.

[22] Vílchez, L. (2014). Entrega de materiales de protección. Huancayo.

[23] Vílchez, L. (2014). Cosecha de maca. Huancayo.

[24] Vílchez, L. (2014). Comercialización de los productos en ferias. Huancayo.

[25] Vílchez, L. (2014). Donación de servicios higiénicos por la certificación de comercio justo. Huancayo.

[26] Vílchez, L. (2015). Beneficios para la comunidad por la certificación de comercio justo. Huancayo.

[27] Vílchez, L. (2016). Tratamiento médico integral por la certificación de comercio justo en Carhuamayo. Huancayo.

[28] Vílchez, L. (2016). Proyecto de secado de maca, vista externa. Huancayo.

[29] Vílchez. L. (2016). Proyecto de secado de maca, vista interna. Huancayo.

[30] World Fair Trade Organization - WFTO. (2017). Principios del comercio justo. Culemborg: WFTO. 